Gd vs dOHb

\title{
Cerebral perfusion imaging: Hypoxia-induced deoxyhemoglobin or gadolinium?
}

\author{
E.S Sayin ${ }^{1,2}$, J. Schulman ${ }^{3,4}$, J. Poublanc ${ }^{5}$, H. Levine ${ }^{1,2}$, L. Venkatraghavan ${ }^{2}$, K. Uludag ${ }^{4,5,6}$, J. \\ Duffin $^{1,2}$, J.A. Fisher* ${ }^{1,2}$, D.J. Mikulis ${ }^{3,4} \&$ O. Sobczyk ${ }^{2,5}$
}

1. Department of Physiology, University of Toronto, Toronto, Ontario, Canada.

2. Department of Anaesthesia and Pain Management, University Health Network, University of Toronto, Toronto, ON, Canada.

3. Department of Medical Biophysics, University of Toronto, Toronto, Ontario, Canada

4. Techna Institute, University Health Network, Toronto, Canada

5. Joint Department of Medical Imaging and the Functional Neuroimaging Lab, University Health Network, Toronto, ON, Canada

6. Center for Neuroscience Imaging Research, Institute for Basic Science \& Department of Biomedical Engineering, Sungkyunkwan University, Suwon, Republic of Korea 
Gd vs dOHb

\begin{abstract}
Assessment of resting cerebrovascular perfusion measures (mean transit time, cerebral blood flow and cerebral blood volume) with magnetic resonance imaging currently requires the intravascular injection of the dynamic susceptibility contrast agent gadolinium. An initial comparison between hypoxia-induced deoxyhemoglobin and gadolinium was made for these measures in six healthy participants. A bolus of deoxyhemoglobin is generated in the lung via transient hypoxia induced by an available computer-controlled gas blender technology employing sequential gas delivery (RespirAct $\left.{ }^{\mathrm{TM}}\right)$. We hypothesised and confirmed perfusion measures from both susceptibility contrast agents would yield similar spatial patterns of cerebrovascular perfusion measures. We conclude that hypoxia-induced deoxyhemoglobin, an endogenously, non-invasively generated, non-allergenic, non-toxic, recyclable, environmentally innocuous molecule, may be suitable to become the first new magnetic resonance imaging susceptibility contrast agent introduction since gadolinium.
\end{abstract}


Gd vs dOHb

\section{Introduction}

Many common conditions such as cigarette smoking, high blood cholesterol, obesity, sedentary lifestyle, diabetes, hypertension, and aging result in silently accumulating cerebrovascular pathologies, for example small vessel disease, venous collagenosis, chronic inflammation and multiple subcortical infarcts. The accumulation of cerebral vascular disease leads to cognitive decline and can precipitate sudden strokes ${ }^{1}$. The health of cerebral perfusion can be assessed by magnetic resonance imaging (MRI)-based perfusion measures such as mean transit time (MTT), cerebral blood volume (CBV) and cerebral blood flow (CBF). In contrast to flow measures, surrogate measures such as those obtained from cognitive tests ${ }^{1}$ may be affected by vascular function, but are not a direct reflection of it.

MRI-based measures of cerebral vascular perfusion measure require an intravascular injection of a dynamic susceptibility contrast agent. Currently gadolinium (Gd) is the only option, but is invasive, expensive ${ }^{2}$, leaks into the extracellular fluid ${ }^{3}$, accumulates in tissue ${ }^{4}$, has risks of causing harm $^{5-7}$, and is an emerging environmental pollutant ${ }^{8}$. Consequently, there remains an urgent world-wide need for an MRI contrast agent that is non-invasive, economical, remains in the blood pool, does not accumulate in the body, is abundant, safe, and is environmentally innocuous. These challenging requirements may be addressed by hypoxia-induced deoxyhemoglobin $(\mathrm{dOHb})^{9,10}$.

Several a-priori considerations prompted the exploration of $\mathrm{dOHb}$ as a potentially useful susceptibility contrast agent. First, although oxyhemoglobin is diamagnetic, it becomes paramagnetic after giving up its oxygen to become $\mathrm{dOHb}^{11}$. Thus, like $\mathrm{Gd}, \mathrm{dOHb}$ changes the 
Gd vs dOHb

homogeneity of a magnetic field inside and outside the blood vessels in proportion to its concentration $([\mathrm{dOHb}])^{12}$. Second, dOHb can be rapidly formed in the blood of pulmonary capillaries by breathing hypoxic gas as previously shown by $\mathrm{Vu}$ et $\mathrm{al}^{13}$ and this process can be rapidly reversed by restoring normoxia by a single breath containing normal levels of oxygen ${ }^{10}$. Third, the limiting factors in the rate of formation and clearing of $\mathrm{dOHb}$ are those in changing the end tidal partial pressure of oxygen $\left(\mathrm{P}_{\mathrm{ET}} \mathrm{O}_{2}\right)$ in the lung. We have developed considerable expertise in inducing rapid, controlled changes in the $\mathrm{P}_{\mathrm{ET}} \mathrm{O}_{2}$ using techniques described as prospective end tidal targeting ${ }^{14,15}$ and sequential gas delivery (SGD) $)^{16,17}$. Finally, at 3 Tesla, $\sim 25 \% \mathrm{dOHb}$ in arterial blood provides a $\mathrm{T} 2 *$ signal change sufficient to generate calculations of MTT, CBV and CBF whose magnitudes and distributions are consistent with those previously published for Gd-based perfusion imaging ${ }^{10}$.

Here we describe the use of hypoxia-induced $\mathrm{dOHb}$ as a susceptibility contrast agent. We use the ensuing changes in cerebral blood oxygen level dependent (BOLD) signal to calculate cerebrovascular MTT, CBV and CBF. To initially evaluate whether dOHb would be a suitable contrast agent for computing perfusion measures, we compare these measures to those calculated from an intravenous injection of $\mathrm{Gd}$ in the same 6 healthy participants. We hypothesize that the relative magnitude and distribution of the perfusion measures calculated using $\mathrm{dOHb}$ as a contrast agent will be similar to those generated using Gd.

\section{Methods}

Participant and Ethics Approval

This study conformed to the standards set by the latest revision of the Declaration of Helsinki 


\section{Gd vs dOHb}

and was approved by the Research Ethics Board of University Health Network according to the guidelines of Health Canada. All participant data was anonymized according to institutional protocols. We recruited 6 participants $(5 \mathrm{M})$ between the ages of 23 and 60 by advertisement and word of mouth. All participants provided written and informed consent prior to scanning. All were healthy individuals, non-smokers, not taking any medications, and with no history of neurological, cardiovascular or kidney diseases.

\section{Respiratory Protocol}

A computerized sequential gas delivery system, (RespirAct ${ }^{\mathrm{TM}}$, Thornhill Medical, Toronto, Canada) was used to control and manipulate $\mathrm{P}_{\mathrm{ET}} \mathrm{O}_{2}$ while maintaining normocapnia, independent of participant ventilation ${ }^{18,19}$. Participants breathed through a facemask sealed to the face with skin tape (Tegaderm, 3M, Saint Paul, MN, U.S.A.) to exclude all but system-supplied gas. The programmed $\mathrm{P}_{\mathrm{ET}} \mathrm{O}_{2}$ stimulus pattern was 4-minutes and 20 seconds long (Fig. 1), and consisted of a $60 \mathrm{~s}$ baseline $\mathrm{P}_{\mathrm{ET}} \mathrm{O}_{2}$ of $95 \mathrm{mmHg}$ (normoxia), a step decrease in $\mathrm{P}_{\mathrm{ET}} \mathrm{O}_{2}$ to $40 \mathrm{mmHg}$ (hypoxia) for 60 seconds, a return to normoxia for 20 seconds, a step decrease in $\mathrm{P}_{\mathrm{ET}} \mathrm{O}_{2}$ to 40 $\mathrm{mmHg}$ for 60 seconds, followed by a return to normoxia for $60 \mathrm{~s}$. Arterial [dOHb] was calculated from $\mathrm{P}_{\mathrm{ET}} \mathrm{O}_{2}$ using the Hill equation describing the in-vivo oxyhemoglobin dissociation curve $\mathrm{e}^{20}$.

\section{MRI Scanning Protocol}

The study was performed using a 3-Tesla scanner (HDx Signa platform, GE healthcare, Milwaukee, WI, USA) with an 8-channel head coil. The experimental protocol consisted of a high-resolution T1-weighted scan followed by two BOLD sequence scans on all participants. The first BOLD scan sequence was acquired during $\mathrm{P}_{\mathrm{ET}} \mathrm{O}_{2}$ manipulation, while the second one 
Gd vs dOHb

was acquired during an injection of Gd. The high-resolution T1-weighted scan was acquired using a 3D spoiled gradient echo sequence with the following parameters: $\mathrm{TI}=450 \mathrm{~ms}$, TR 7.88 $\mathrm{ms}, \mathrm{TE}=3 \mathrm{~ms}$, flip angle $=12^{\circ}$, voxel size $=0.859 \times 0.859 \times 1 \mathrm{~mm}$, matrix size $=256 \times 256,146$ slices, field of view $=24 \times 24 \mathrm{~cm}$, no interslice gap. The BOLD data was acquired using a T2*_ weighted gradient echoplanar imaging sequence with the following parameters: TR = $1500 \mathrm{ms,}$ $\mathrm{TE}=30 \mathrm{~ms}$, flip angle $=73^{\circ}, 29$ slices voxel size $=3 \mathrm{~mm}$ isotropic voxels and matrix size $=64 \times$ 64. After the completion of $\mathrm{P}_{\mathrm{ET}} \mathrm{O}_{2}$ targeting protocol, the participant returned to free breathing on room air for at least 5 minutes before the Gd based perfusion acquisition for which Gadovist, 5 $\mathrm{ml}$ was injected intravenously at a rate of $5 \mathrm{ml} / \mathrm{s}$ followed by $30 \mathrm{ml}$ of saline at a rate of $5 \mathrm{ml} / \mathrm{s}$.

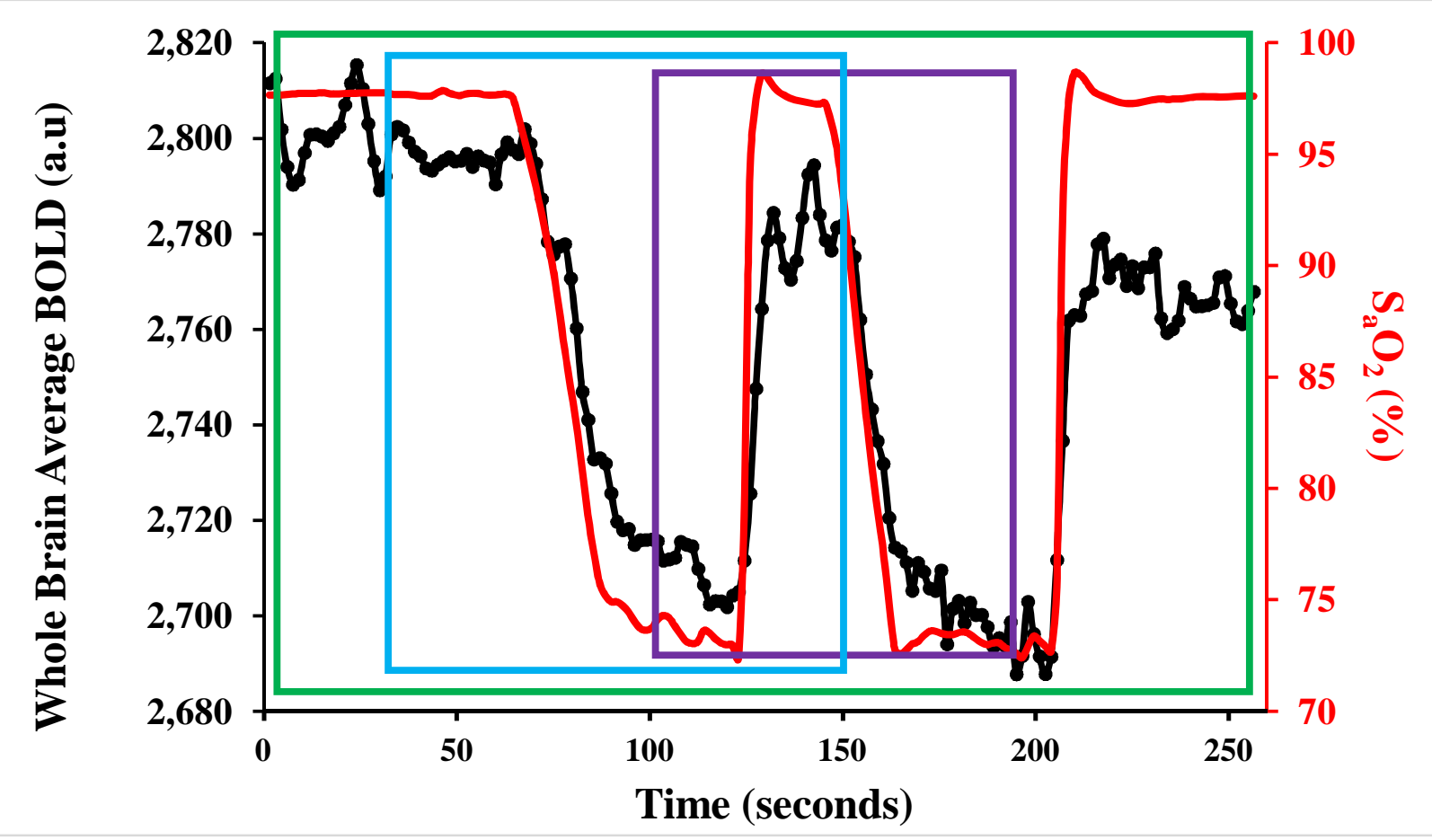

Figure 1. The test protocol in a representative participant. The arterial $\mathrm{O}_{2}$ saturation $\left(\mathrm{S}_{\mathrm{a}} \mathrm{O}_{2}\right)$ (red), calculated from the $\mathrm{P}_{\mathrm{ET}} \mathrm{O}_{2}$ and the oxygen dissociation curve ${ }^{20}$, and the whole brain average BOLD response (black) in arbitrary units (a.u). The test protocol was divided into selected parts for separate analyses: "single hypoxic" (blue rectangle), "double hypoxic" (green rectangle) and "single normoxic" (purple rectangle). 
Gd vs dOHb

\section{Data Analysis}

Basics

MR images generated by changes in $\mathrm{P}_{\mathrm{ET}} \mathrm{O}_{2}$ data were analyzed using Analysis of Functional Neuroimaging (AFNI) software (National Institutes of Health, Bethesda, Maryland) ${ }^{21}$. BOLD images were volume registered, slice-time corrected and co-registered to the anatomical images. The T1-weighted spoiled gradient images were segmented into gray (GM) and white matter (WM) using Statistical Parametric mapping 8 (SPM8) (Wellcome Department of Imaging Neuroscience, Institute of Neurology, University College, London, UK).

\section{Determining the BOLD signal change and the Contrast to Noise Ratio}

The BOLD signal change ( $\triangle \mathrm{BOLD})$ and contrast to noise ratio $(\mathrm{CNR})$ was calculated for Gd and each selected part of the protocol. To examine the effectiveness of using different parts of the $\mathrm{dOHb}$ protocol, calculations were repeated using each selected part of the protocol outlined in Fig. 1.

To calculate CNR, a 20s continuous baseline signal at the beginning or end of each dataset was selected. A linear regression between each voxel BOLD signal and the average whole brain BOLD signal was calculated. The slope of the regression multiplied by the average whole brain BOLD signal peak-to-peak was used to calculate relative $\triangle B O L D$ for each voxel of the brain. CNR was calculated by dividing $\triangle B O L D$ by the standard deviation of the linear regression residuals $^{10}$. Average $\mathrm{CNR}$ and $\triangle \mathrm{BOLD}$ values for GM, WM and GM/WM ratios were calculated for each participant.

\section{Determining the arterial input function for $\mathrm{dOHb}$}

Perfusion measures for $\mathrm{dOHb}$ were calculated using two separate global arterial input functions 


\section{Gd vs dOHb}

(AIF): the measured BOLD signal over the middle cerebral artery (AIF-MCA) and the $\mathrm{SaO}_{2}$ $\left(\mathrm{AIF}-\mathrm{SaO}_{2}\right.$ ) calculated from the $\mathrm{P}_{\mathrm{ET}} \mathrm{O}_{2}$. Identifying the AIF from a voxel over the MCA is fraught $^{22}$; however, we expended our best efforts to optimize the choice of voxel. Smoothing was applied to each voxel in the dataset using an adaptive mean filter of width $(n=7)$ using AFNI software. FSL tool VERBENA software ${ }^{23}$ was used to calculate MTT and relative CBF (rCBF) using the Gd data and the data from the double hypoxic protocol using each AIF. Relative CBV (rCBV) was calculated as MTT multiplied by rCBF. Maps of the $\mathrm{dOHb}$ and $\mathrm{Gd}$ perfusion measures were generated using AFNI software and overlayed onto their respective anatomical images. To normalize for $\mathrm{rCBF}$ and $\mathrm{rCBV}$ perfusion maps within participants for $\mathrm{Gd}, \mathrm{dOHb}$ (MCA), dOHb ( $\mathrm{SaO} 2)$, the ratio between whole brain average $\mathrm{Gd}$ and the respective whole brain average for each $\mathrm{dOHb} \mathrm{AIF}$ were calculated. Furthermore, the $\mathrm{dOHb}$ perfusion maps were scaled with the calculated ratio to normalize the relative measures within participants. Average MTT values (the only absolute measure) for GM, WM, and GM/WM ratios were calculated for each participant.

\section{Statistical Analysis}

Statistical comparisons within participants for CNR and $\triangle \mathrm{BOLD}$ values across all methods (Gd and each protocol) for GM, WM and GM/WM ratios were performed using a one-way repeated measures analysis of variance (rmANOVA) with a Holm-Sidak method of multiple comparisons correction $(\alpha=0.05)$. In the event of an Equal Variance test or a Normality Test (Shapiro-Wilk) failure, a Friedman Repeated Measures Analysis of Variance on Ranks was used with Tukey Test method of multiple comparisons correction. A rmANOVA was performed on the average MTT values for GM, WM and GM/WM ratios between Gd (AIF-MCA), dOHb (AIF-MCA) and $\mathrm{dOHb}\left(\mathrm{AIF}-\mathrm{SaO}_{2}\right)$ for each participant $(\alpha=0.05)$. 
Gd vs dOHb

\section{Results}

The Hypoxic Stimulus

The brief ( $60 \mathrm{~s}$ ) hypoxia induced in these tests was well tolerated by all participants. After completion of the test, only one of the participants reported having had moderate shortness of breath.

\section{The BOLD Signal}

The $\triangle \mathrm{BOLD}$ and CNR for WM and GM are presented in Table 1 for Gd and the three selected parts of the protocol: single hypoxic, double hypoxic, and single normoxic. Fig. 2 displays axial images of $\triangle B O L D$ maps in a representative participant. Although all types of the selected parts of the protocol yielded similar values, the double hypoxic part resulted in the highest $\triangle B O L D$ and CNR (Table 1). Consequently, we chose the double hypoxic part in the calculations of the perfusion measures for comparison to $\mathrm{Gd}$. 
Gd vs dOHb

Table 1. Average (SD) measures of $\triangle B O L D$ and CNR calculated for Gd, and the three selected parts of the protocol: single hypoxic, double hypoxic, and single normoxic for the six participants.

\begin{tabular}{|c|c|c|c|c|c|c|}
\hline & \multicolumn{3}{|c|}{$\triangle B O L D$} & \multicolumn{3}{|c|}{ CNR } \\
\hline & GM & WM & GM/WM & GM & WM & GM/WM \\
\hline Gd & $48.38(13.22)$ & $14.91(4.35)$ & 3.25 & $22.56(4.85)$ & $12.19(3.36)$ & 1.85 \\
\hline Single Hypoxic & $7.28(2.42)$ & $2.99(0.95)$ & 2.43 & $4.54(1.28)$ & $2.57(0.76)$ & 1.77 \\
\hline $\begin{array}{l}\text { Double } \\
\text { Hypoxic }\end{array}$ & $9.31(2.79)$ & $3.60(0.95)$ & 2.58 & $5.38(1.68)$ & $3.16(1.01)$ & 1.70 \\
\hline $\begin{array}{l}\text { Single } \\
\text { Normoxic }\end{array}$ & $8.65(2.67)$ & $3.53(1.02)$ & 2.11 & $4.91(1.46)$ & $3.04(1.01)$ & 1.61 \\
\hline
\end{tabular}

GM Gd CNR was significantly different from those of all parts of the protocol $(P<0.001)$, however no significant differences found among the parts (P>0.8). WM GdCNR was significantly different from those of the single hypoxic and normoxic parts $(P<0.05)$ but not the double hypoxic part $(P>0.05)$. The only significant difference in CNR was found in the GM/WM ratio between $G d$ and that of the single normoxic part $(P<0.05)$. The GM $\triangle B O L D G d$ was significantly different from those of all parts $(P<0.001)$ which did not differ among themselves $(P>0.8)$. In addition, WM $\triangle B O L D G d$ was significantly different from those of all parts $(P<0.001)$ however no significant difference was found between parts $(P>0.8)$. The only significant difference in $\triangle B O L D$ GM/WM ratio was between $G d$ and the single hypoxic and normoxic parts $(P<0.05)$. 


\section{Gd}

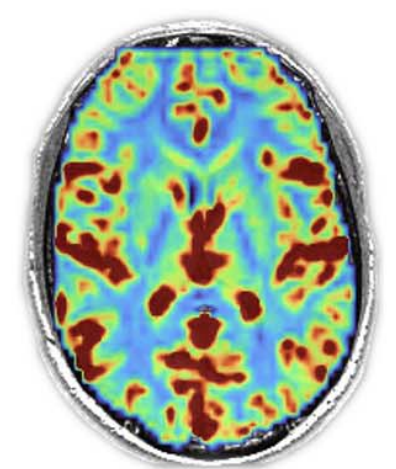

\% BOLD

\section{Single Hypoxic Double Hypoxic}
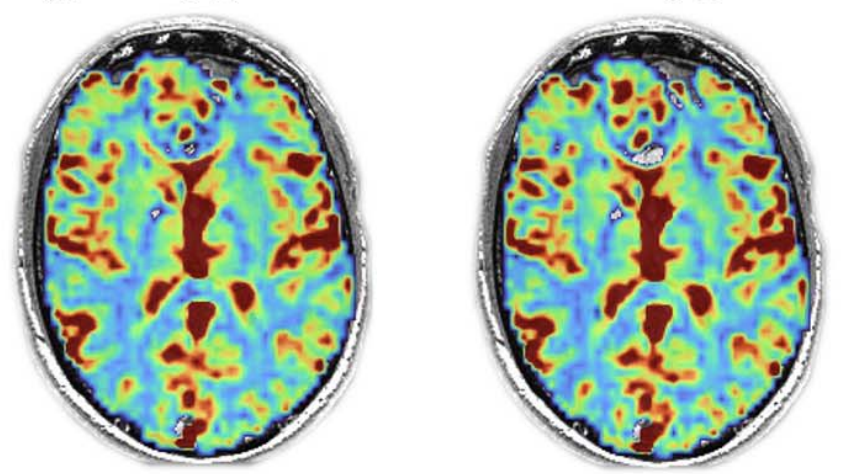

\% BOLD
Single Normoxic

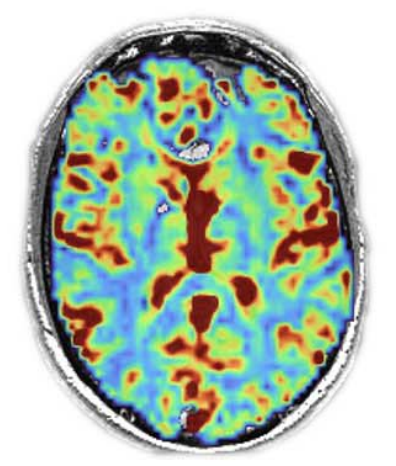

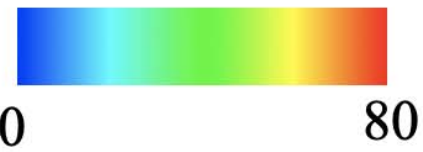

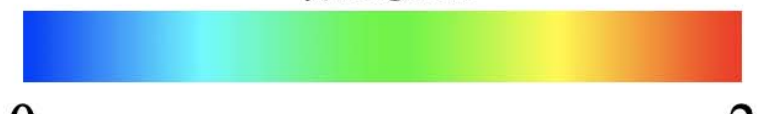

0

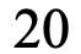

Figure 2. The $\triangle B O L D$ maps for a representative participant comparing Gd and selected parts of the protocol: single hypoxic, double hypoxic, and single normoxic. Note: If the difference in signal strength is accounted for, all the maps appear similar.

Determining the AIF

The perfusion measures, MTT, rCBF and rCBV, were calculated for Gd using an AIF from the MCA. Similarly, they were calculated for the $\mathrm{dOHb}$ double hypoxic part using an AIF from both the $\mathrm{MCA}$ and $\mathrm{SaO}_{2}$. Fig. 3 displays perfusion maps for a representative participant. The perfusion maps for all participants can be found in the supplementary data (Fig. S1). 


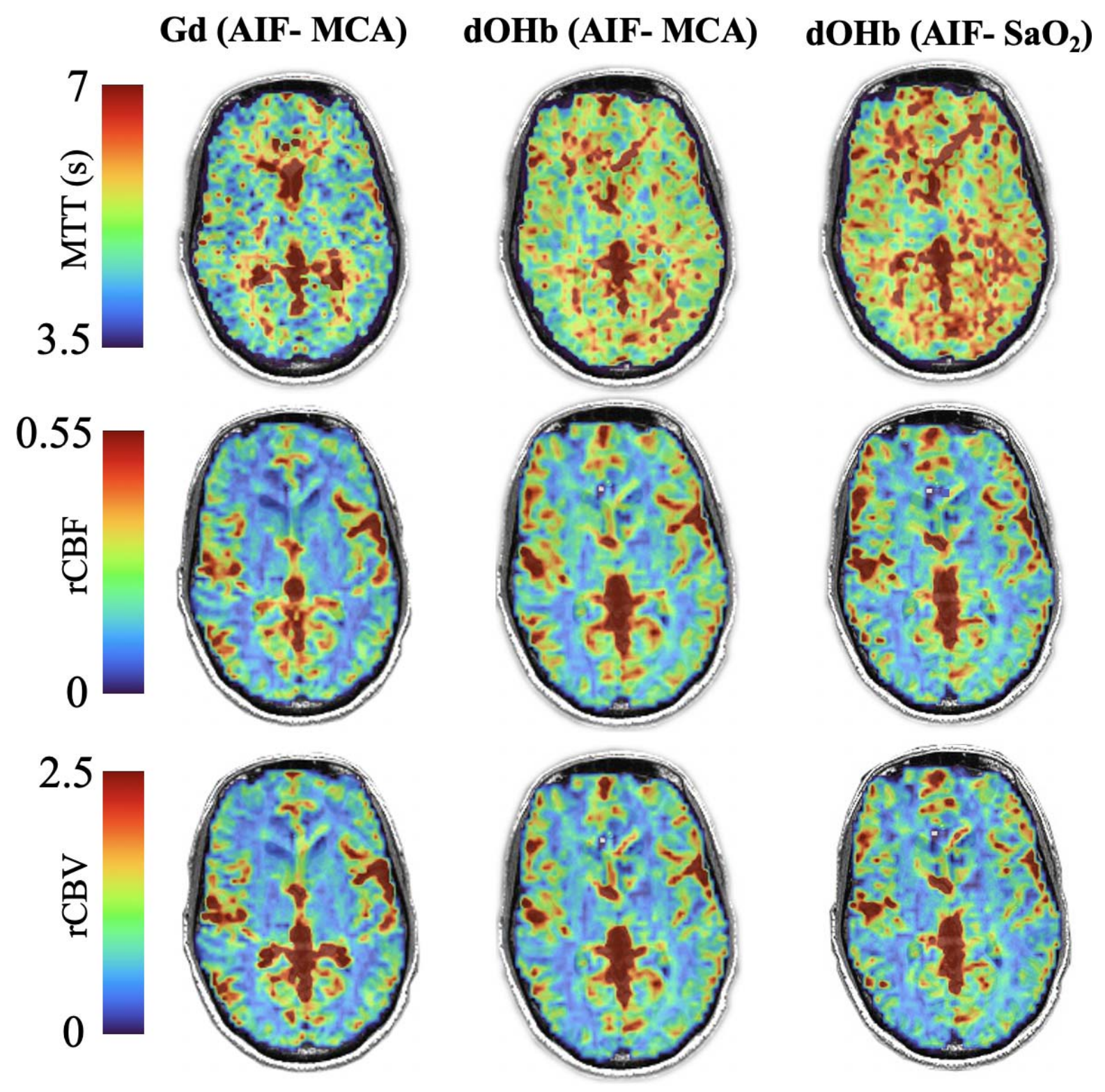

Figure 3. Perfusion maps of MTT, $\mathrm{rCBF}$ and $\mathrm{rCBV}$ of a representative participant comparing dOHB: AIF-MCA and AIF- $\mathrm{S}_{\mathbf{a}} \mathrm{O}_{2}$ to Gd. Note: The spatial distribution of dOHb AIF-MCA and AIF-SaO 2 are similar to each other and Gd AIF-MCA. 
Gd vs dOHb

MTT

The average MTT, the only measure in absolute units, in GM and WM for Gd and the double hypoxic part of the protocol using both the MCA and $\mathrm{SaO}_{2}$ AIF in 6 participants is displayed in

Fig. 4. There were no significant within-participant differences in MTT GM/WM ratio ( $\mathrm{p}=0.282$,

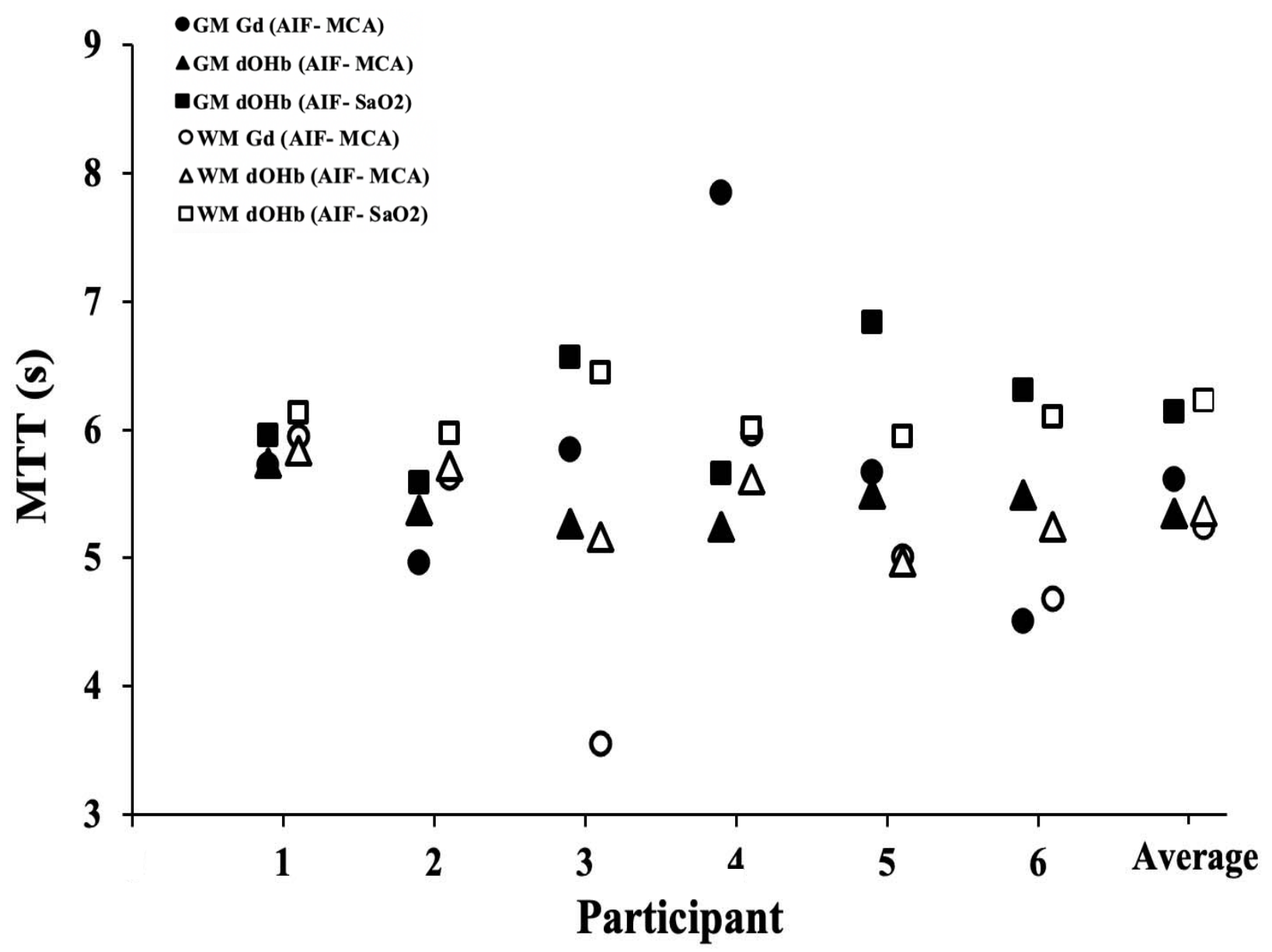

Figure 4. MTT values (s) for Gd and dOHb in GM and WM for the 6 participants. The average values are shown on the right. A rmANOVA found that MTT measures within each participant were not significantly different in GM $(\mathrm{P}=0.325)$. The following comparisons were not significantly different: WM, MTT measures calculated for dOHb (AIF-MCA) and (AIF-SaO2) (P $=0.177)$; MTT measures for Gd (AIF-MCA) and dOHb (AIF-MCA) $(\mathrm{P}=0.384)$. However, within participant MTT differences between Gd (AIF-MCA) and dOHb $\left(\mathrm{AIF} \mathrm{SaO}_{2}\right)$ were small but statistically significant $(\mathrm{P}=0.038)$.

$\alpha=0.05)$. 
Gd vs dOHb

\section{Discussion}

\section{Main finding}

The main finding of this preliminary study is that $\mathrm{dOHb}$ used as a contrast agent displayed similar pattern and spatial distribution in maps of MTT, rCBV and rCBF to those generated by Gd. Currently, Gd is the only option for consistent susceptibility-based contrast imaging. By comparing perfusion measures obtained using $\mathrm{Gd}$ and $\mathrm{dOHb}$ in the same participants under uniform protocols we sought to control for methodological, temporal, and individual participant variables, and thereby isolate differences in results as indicators of inherent differences between the two methods. Despite minimal effort at optimization of dOHb-based data, these differences were judged to be small and justify follow-up investigation. The overarching aim would be to recruit $\mathrm{dOHb}$ contrast as a non-invasive, repeatable, rapid, direct measure of resting cerebrovascular perfusion suitable for monitoring the status and progress of cerebrovascular pathophysiology underlying condition such as cognitive dysfunction and stroke.

\section{Hypoxia tolerance and safety}

In designing this protocol, we estimated that a physiologically tolerable arterial $\mathrm{PO}_{2}$ of about 40 $\mathrm{mmHg}$ would produce a $[\mathrm{dOHb}]$ change of about $25 \%{ }^{20}$ which would generate a sufficient change in the BOLD signal at $3 \mathrm{~T}$ to test the value of $\mathrm{dOHb}$ as a susceptibility contrast agent ${ }^{10}$. To put the hypoxia used in these tests into perspective, a study of the cerebral blood flow effects of hypoxia reported that test participants had no distress and negligible changes in respiratory rate and heart rate while $\mathrm{PO}_{2}$ was maintained at about $50 \mathrm{mmHg}$ for up to $20 \mathrm{~min}^{24}$. Rebreathing tests at isoxic $\mathrm{P}_{\mathrm{ET}} \mathrm{O}_{2}=40$ and $50 \mathrm{mmHg}$ are routinely employed for assessing respiratory 
Gd vs dOHb

chemoreflexes $^{25}$ even in heart failure patients ${ }^{26}$. Finally, we note that some $20-40$ million Americans suffer from moderate to severe obstructive sleep apnea, many of whom also have severe comorbidities including cerebral and myocardial ischemia ${ }^{27}$, and sleep studies have recorded the frequent occurrence of obstructions where $\mathrm{P}_{\mathrm{ET}} \mathrm{O}_{2}$ falls into the $20-40 \mathrm{mmHg}$ $\operatorname{range}^{28}$

\section{Methodological Considerations}

This study followed the recently described findings of Coloigner ${ }^{29}$ and $\mathrm{Vu}^{13}$ who demonstrated that abrupt increases in arterial $[\mathrm{dOHb}]$, induced by transient hypoxia, produce measurable changes in the BOLD signal. Their reports noted the similarity of perfusion measures using $\mathrm{dOHb}$ as a contrast agent to historical values but did not validate the relationship by comparison to measures using $\mathrm{Gd}$. Abrupt transient changes in [ $\mathrm{dOHb}$ ] have not otherwise been considered as a susceptibility contrast agent due to the complicated nature of precisely controlling and inducing abrupt targeted changes in $\mathrm{P}_{\mathrm{ET}} \mathrm{O}_{2}$ in individuals. Vu and colleagues ${ }^{13}$ used inhaled nitrogen, a popular technique to produce a transient hypoxia ${ }^{30}$. However, when using nitrogen as the source gas, and a $2 \mathrm{~L}$ reservoir on the inspiratory limb to buffer the progress of hypoxia, the duration and magnitude of the reduction of $\mathrm{P}_{\mathrm{ET}} \mathrm{O}_{2}$ become highly sensitive to the pattern and extent of breathing, and therefore lacked precise control ${ }^{14}$.

In this study, we examined the CNR for the three selected parts of the protocol. Previously, Poublanc et $a 1^{10}$ determined that CNR increased with repeated hypoxic stimuli but minimal improvement after a second hypoxic stimulus. The results from our experiments showed that the double hypoxic part of the protocol yielded a slightly larger CNR compared to the single hypoxic or the single normoxic parts (Table 1), consequently that was the test protocol used for 
Gd vs dOHb

comparison to Gd. The differences between the three protocols were small indicating that further studies may yield a suitable compromise between a simpler protocol and CNR.

For Gd, abrupt changes in concentration are attempted by rapid intravenous injection at $5 \mathrm{ml} / \mathrm{s}$ followed by a bolus of saline. Nevertheless, the contrast is dispersed as it passes the right heart, loses contrast in the lungs and mixes with the blood in the left heart. To approximate a squarewave leading edge for a $\mathrm{dOHb}$ bolus it is necessary to implement a rapid change of $\mathrm{PETO}_{2}$ in the lung, which is resisted by the dispersing effects of the inspired gases progressively diluting and replacing those in the lungs of participants. The approach used here to induce a targeted level of hypoxia was to pre-calculate and administer the optimum level of nitrogen and oxygen for each successive breath to most efficiently attain and maintain a targeted $\mathrm{PETO}_{2}$, taking into account the lung volume, oxygen consumption, and breath size. We emphasize that the great advantage of these methods is that the calculations are made prospectively and do not rely on feedback loops, so that the trajectory to the target $\mathrm{PETO}_{2}$ is optimized with each breath ${ }^{14}$. The delivery of the optimized gases is automatically synchronized to the particular breathing pattern of the participant by an automated gas blender (RespirAct $\left.{ }^{\mathrm{TM}}\right)$ implementing sequential gas delivery ${ }^{17}$. The rapidly formed $\mathrm{dOHb}$ in the pulmonary capillaries traverses only the left atrium and left ventricle, minimizing the opportunity for dispersion in the blood before passing into the cerebral arteries. We suggest this accounts the high similarity of the perfusion measures calculated from AIF's using $\mathrm{P}_{\mathrm{ET}} \mathrm{O}_{2}$ and voxel BOLD signal over the MCA.

\section{Differences between $\mathrm{Gd}$ and $\mathrm{dOHb}$}

As an intravascular paramagnetic molecule, $\mathrm{dOHb}$ has several characteristics that differ from those of Gd (Supplementary Data Table 1). First, while Gd distributes throughout the plasma, 
Gd vs dOHb

$\mathrm{dOHb}$ is confined inside red blood cells. One consequence of this differing distribution is that $[\mathrm{Gd}]$ increases as the hematocrit falls in the capillaries while [dOHb] increases ${ }^{31}$. These effects have implications as to the relationship between signal size and contrast concentration ${ }^{32}$. A second consequence is that Gd passes readily out of capillaries into tissues such as the lungs resulting in dispersal before arrival in the arteries. In the brain, Gd is contained intravascularly by the blood-brain barrier making it a good marker for its integrity, for example, in brain tumors $^{33}$. By contrast, dOHb does not leak into tissues and remains intravascular regardless of the blood-brain barrier unless there is active bleeding. Second, the arterial [Gd] varies with dose $^{34}$, speed of injection ${ }^{35}$, cardiac output ${ }^{36}$, degree of dispersion ${ }^{37}$, and amount leaked from the vessels prior to arrival at the arteries ${ }^{38}$. Of these, only dose and speed of injection can be controlled, so that it is not possible to deliberately implement a particular concentration or profile of [Gd] as an AIF. An AIF may be measured at the MCA, but this measurement is also problematic $^{39,40}$, and in most cases must be deconvolved from the residue function. Whereas the exact $[\mathrm{dOHb}]$ and its profile corresponds to that of $\mathrm{P}_{\mathrm{ET}} \mathrm{O}_{2}$. It is independent of cardiac output and existing leaks into interstitial spaces. Finally, for Gd, calculations of the cerebral perfusion measures must be measured on the first pass of $\mathrm{Gd}$ as some of the molecules traversing high flow short distance organs such as thyroid, heart and bronchial circulation may recirculate quickly ${ }^{35}$, with its arrival potentially overlapping the AIF. Gd is only slowly cleared from circulation, thereby confounding further measures for about 2 hours ${ }^{41}$. By contrast, all changes in dOHb are oxidized when $\mathrm{P}_{\mathrm{ET}} \mathrm{O}_{2}$ is restored to normoxia, enabling multiple repeated measures at close time intervals. 
Gd vs dOHb

\section{Limitations}

Optimization of the $\mathrm{dOHb}$ method is ongoing. We believe that the selected hypoxic stimulus pattern used in this study generated an adequate bolus of contrast as indicated by the agreement of the $\mathrm{dOHb}$ perfusion maps with those generated using $\mathrm{Gd}$. However, further development is needed to determine the optimal $[\mathrm{dOHb}]$ and the AIF pattern for studies of people with healthy vasculature, as well as those with pathology. The scan sequence parameters need to be optimized for whole brain coverage, temporal resolution (TR) and thereby CNR. In addition, the selection of the AIF in an MCA voxel compared to that using the $\mathrm{SaO}_{2}$ requires further investigation.

\section{Future Directions}

For this study we chose a particular hypoxic stimulus pattern to induce the $\mathrm{dOHb}$ changes, and magnetic resonance scanning settings that we felt were appropriate. However, these choices may be optimised after further experimentation. We compared perfusion measures between $\mathrm{dOHb}$ and Gd, but the considered gold-standard for such measures uses positron emission tomography (PET). Comparisons of Gd and PET show only moderate consistency ${ }^{42-44}$. Nevertheless, it would be instructive to compare the maps of perfusion measures against this gold-standard, particularly in patients with cerebrovascular disease. This study was an initial comparison of a new susceptibility molecule to a well-known clinical standard. Larger studies are required to identify normal ranges of perfusion measures using $\mathrm{dOHb}$ as a contrast agent in population pools divided by age, sex, underlying diseases, and specific cerebrovascular pathophysiology. 
Gd vs dOHb

\section{Conclusion}

In conclusion, using $\mathrm{dOHb}$ as a contrast agent provided similar set of perfusion maps and measures to those of $\mathrm{Gd}$ in the same healthy individuals. This finding supports the continued exploration of $\mathrm{dOHb}$ as a finely controlled endogenous contrast agent with the advantages of being an endogenous, non-invasively generated, non-allergenic, non-toxic, recyclable, environmentally innocuous molecule. It may be the first new MRI susceptibility contrast agent introduction since gadolinium.

\section{Data Availability}

Anonymized data will be shared by request from any qualified investigator for purposes such as replicating procedures and results presented in the article provided that data transfer is in agreement with the University Health Network and Health Canada legislation on the general data protection regulation.

\section{References}

1 Heshmatollah, A. et al. Long-term trajectories of decline in cognition and daily functioning before and after stroke. Journal of neurology, neurosurgery and psychiatry, doi:10.1136/jnnp-2021-326043 (2021).

2 Nunn, A. D. The Cost of Developing Imaging Agents for Routine Clinical Use. Investigative radiology 41, 206-212, doi:10.1097/01.rli.0000191370.52737.75 (2006).

3 Quarles, C. C., Bell, L. C. \& Stokes, A. M. Imaging vascular and hemodynamic features of the brain using dynamic susceptibility contrast and dynamic contrast enhanced MRI. NeuroImage (Orlando, Fla.) 187, 32-55, doi:10.1016/j.neuroimage.2018.04.069 (2019). 
4 Kanda, T., Ishii, K., Kawaguchi, H., Kitajima, K. \& Takenaka, D. High Signal Intensity in the Dentate Nucleus and Globus Pallidus on Unenhanced T1-weighted MR Images: Relationship with Increasing Cumulative Dose of a Gadolinium-based Contrast Material. Radiology 270, 834-841, doi:10.1148/radiol.13131669 (2014).

5 Li, A., Wong, C. S., Wong, M. K., Lee, C. M. \& Au Yeung, M. C. Acute adverse reactions to magnetic resonance contrast media gadolinium chelates. British journal of radiology 79, 368-371, doi:10.1259/bjr/88469693 (2006).

6 Gulani, V. et al. Gadolinium deposition in the brain: summary of evidence and recommendations. Lancet Neurol 16, 564-570, doi:10.1016/S1474-4422(17)30158-8 (2017).

7 Runge, V. M. Safety of approved MR contrast media for intravenous injection. Journal of magnetic resonance imaging 12, 205-213, doi:10.1002/15222586(200008)12:2<205::AID-JMRI1>3.0.CO;2-P (2000).

8 Le Goff, S. et al. Compound-specific recording of gadolinium pollution in coastal waters by great scallops. Scientific reports 9, 8015-8015, doi:10.1038/s41598-019-44539-y (2019).

9 Chai, Y. et al. White matter has impaired resting oxygen delivery in sickle cell patients. Am J Hematol 94, 467-474, doi:10.1002/ajh.25423 (2019).

10 Poublanc, J. et al. Perfusion MRI using endogenous deoxyhemoglobin as a contrast agent: Preliminary data. MRM (2021).

11 Pauling, L. The diamagnetic anisotropy of aromatic molecules. The Journal of chemical physics 4, 673-677, doi:10.1063/1.1749766 (1936). 
Gd vs dOHb

12 Blockley, N. P. et al. Field strength dependence of R1 and R2 relaxivities of human whole blood to ProHance, vasovist, and deoxyhemoglobin. Magnetic resonance in medicine 60, 1313-1320, doi:10.1002/mrm.21792 (2008).

$13 \mathrm{Vu}, \mathrm{C}$. et al. Quantitative perfusion mapping with induced transient hypoxia using BOLD MRI. Magnetic resonance in medicine 85, 168-181, doi:10.1002/mrm.28422 (2021).

14 Slessarev, M. et al. Prospective targeting and control of end-tidal CO2 and $\mathrm{O} 2$ concentrations. J Physiol 581, 1207-1219 (2007).

15 Ito, S. et al. Non-invasive prospective targeting of arterial PCO2 in subjects at rest. $J$ Physiol 586, 3675-3682 (2008).

16 Sommer, L. Z. et al. A simple breathing circuit minimizing changes in alveolar ventilation during hyperpnoea. Eur Respir J 12, 698-701 (1998).

17 Fisher, J. A., Iscoe, S. \& Duffin, J. Sequential gas delivery provides precise control of alveolar gas exchange. Respir Physiol Neurobiol 225, 60-69, doi:10.1016/j.resp.2016.01.004 (2016).

18 Fisher, J. A. The CO2 stimulus for cerebrovascular reactivity: Fixing inspired concentrations vs. targeting end-tidal partial pressures. Journal of Cerebral Blood Flow \& Metabolism 36, 1004-1011, doi:10.1177/0271678x16639326 (2016).

19 Fierstra, J. et al. Measuring cerebrovascular reactivity: what stimulus to use? J Physiol 591, 5809-5821, doi:10.1113/jphysiol.2013.259150 (2013).

20 Balaban, D. Y. et al. The in-vivo oxyhaemoglobin dissociation curve at sea level and high altitude. Respir Physiol Neurobiol 186, 45-52, doi:10.1016/j.resp.2012.12.011 (2013). 


\section{Gd vs dOHb}

21 Cox, R. W. AFNI: software for analysis and visualization of functional magnetic resonance neuroimages. Comput Biomed Res 29, 162-173 (1996).

22 Calamante, F. Arterial input function in perfusion MRI: A comprehensive review. Progress in nuclear magnetic resonance spectroscopy 74, 1-32, doi:10.1016/j.pnmrs.2013.04.002 (2013).

23 Chappell, M. A., Mehndiratta, A. \& Calamante, F. Correcting for large vessel contamination in dynamic susceptibility contrast perfusion MRI by extension to a physiological model of the vasculature. Magnetic resonance in medicine 74, 280-290, doi:10.1002/mrm.25390 (2015).

24 Harris, A. D. et al. Cerebral blood flow response to acute hypoxic hypoxia. NMR in biomedicine 26, 1844-1852, doi:10.1002/nbm.3026 (2013).

25 Duffin, J. Measuring the respiratory chemoreflexes in humans. Respiratory Physiology \& Neurobiology 177, 71-79 (2011).

26 Keir, D. A., Duffin, J. \& Floras, J. S. Measuring Peripheral Chemoreflex Hypersensitivity in Heart Failure. Frontiers in physiology 11, 595486-595486, doi:10.3389/fphys.2020.595486 (2020).

27 Punjabi, N. M. The Epidemiology of Adult Obstructive Sleep Apnea. Proceedings of the American Thoracic Society 5, 136-143, doi:10.1513/pats.200709-155MG (2008).

28 Tilkian, A. G. et al. Hemodynamics in sleep induced apnea. Studies during wakefulness and sleep. Annals of internal medicine 85, 714-719, doi:10.7326/0003-4819-85-6-714 (1976). 
Gd vs dOHb

29 Coloigner, J. et al. Transient Hypoxia Model Revealed Cerebrovascular Impairment in Anemia Using BOLD MRI and Near $\square$ Infrared Spectroscopy. Journal of magnetic resonance imaging 52, 1400-1412, doi:10.1002/jmri.27210 (2020).

30 Pfoh, J. R. et al. Comparing and characterizing transient and steady-state tests of the peripheral chemoreflex in humans. Experimental Physiology 101, 432-447, doi:10.1113/EP085498 (2016).

31 Calamante, F. Perfusion MRI using dynamic-susceptibility contrast MRI: Quantification issues in patient studies. Topics in magnetic resonance imaging $\mathbf{2 1}, \mathbf{7 5 - 8 5 ,}$ doi:10.1097/RMR.0b013e31821e53f5 (2010).

32 Bleeker, E. J. W. et al. New criterion to aid manual and automatic selection of the arterial input function in dynamic susceptibility contrast MRI: New Criterion for Arterial Input Function Selection. Magnetic resonance in medicine 65, 448-456, doi:10.1002/mrm.22599 (2011).

33 Boxerman, J. L., Schmainda, K. M. \& Weisskoff, R. M. Relative Cerebral Blood Volume Maps Corrected for Contrast Agent Extravasation Significantly Correlate with Glioma Tumor Grade, Whereas Uncorrected Maps Do Not. American journal of neuroradiology : AJNR 27, 859-867 (2006).

34 Willats, L., Connelly, A. \& Calamante, F. Improved deconvolution of perfusion MRI data in the presence of bolus delay and dispersion. Magnetic resonance in medicine $\mathbf{5 6}$, 146-156, doi:10.1002/mrm.20940 (2006).

35 van Osch, M. J. P. et al. Model of the human vasculature for studying the influence of contrast injection speed on cerebral perfusion MRI. Magnetic resonance in medicine $\mathbf{5 0}$, 614-622, doi:10.1002/mrm.10567 (2003). 
Gd vs dOHb

36 Upton, R. N., Ludbrook, G. L., Grant, C. \& Martinez, A. M. Cardiac Output is a Determinant of the Initial Concentrations of Propofol After Short-Infusion Administration. Anesthesia and analgesia 89, 545-545, doi:10.1097/00000539199909000-00002 (1999).

37 Willats, L. \& Calamante, F. The 39 steps: evading error and deciphering the secrets for accurate dynamic susceptibility contrast MRI: THE 39 STEPS: TOWARDS ACCURATE DSC-MRI. NMR in biomedicine 26, 913-931, doi:10.1002/nbm.2833 (2013).

38 Shiroishi, M. S. et al. Principles of T2-weighted dynamic susceptibility contrast MRI technique in brain tumor imaging. Journal of magnetic resonance imaging 41, 296-313, doi:10.1002/jmri.24648 (2015).

39 Bleeker, E. J. W., van Buchem, M. A. \& van Osch, M. J. P. Optimal location for arterial input function measurements near the middle cerebral artery in first-pass perfusion MRI. Journal of cerebral blood flow and metabolism 29, 840-852, doi:10.1038/jcbfm.2008.155 (2009).

40 van Osch, M. J. P., van der Grond, J. \& Bakker, C. J. G. Partial volume effects on arterial input functions: Shape and amplitude distortions and their correction. Journal of magnetic resonance imaging 22, 704-709, doi:10.1002/jmri.20455 (2005).

41 Levin, J. M. et al. Sequential dynamic susceptibility contrast MR experiments in human brain: Residual contrast agent effect, steady state, and hemodynamic perturbation. Magnetic resonance in medicine 34, 655-663, doi:10.1002/mrm.1910340503 (1995).

42 Grandin, C. B., Bol, A., Smith, A. M., Michel, C. \& Cosnard, G. Absolute CBF and CBV measurements by MRI bolus tracking before and after acetazolamide challenge: 
Gd vs dOHb

repeatabilily and comparison with PET in humans. Neuroimage 26, 525-535, doi:10.1016/j.neuroimage.2005.02.028 (2005).

43 Carroll, T. J. et al. Absolute quantification of cerebral blood flow with magnetic resonance, reproducibility of the method, and comparison with $\mathrm{H} 2(15) \mathrm{O}$ positron emission tomography. Journal of cerebral blood flow and metabolism 22, 1149-1156 (2002).

44 Ostergaard, L. et al. Cerebral blood flow measurements by magnetic resonance imaging bolus tracking: comparison with $[(15) \mathrm{O}] \mathrm{H} 2 \mathrm{O}$ positron emission tomography in humans. $J$ Cereb Blood Flow Metab 18, 935-940, doi:10.1097/00004647-199809000-00002 (1998).

45 Conturo, T. E. et al. Arterial input functions for dynamic susceptibility contrast MRI: Requirements and signal options. Journal of magnetic resonance imaging 22, 697-703, doi:10.1002/jmri.20457 (2005).

\section{Acknowledgements}

All the authors thank Toronto Western Hospital MR technologist Keith Ta for all his help in acquiring the MR imaging data, Abby Skandaraniyam for study coordination and Dr. John Wood for feedback on the final draft of the paper. The study was supported by the Institute for Basic Science, Suwon, Republic of Korea (IBS-R015-D1) to Kamil Uludag.

\section{Author contributions}

All authors contributed to the design and conceptualization of the study. ESS, OS, JD and JP were involved in the data analysis. ESS, OS, JP, drafted the initial draft of the manuscript. All 
Gd vs dOHb

authors participated in multiple rounds of review of data and editing the manuscript. All authors have reviewed and endorse the final draft.

\section{Competing Interest Statement}

JAF and DJM contributed to the development of the automated end-tidal targeting device,

RespirAct $^{\mathrm{TM}}$ (Thornhill Research Inc., TRI) used in this study and have equity in the company.

JAF, OS and JD receive salary support from TRI. TRI provided no other support for the study.

All other authors have no disclosures. 
bioRxiv preprint doi: https://doi.org/10.1101/2021.11.08.467772; this version posted November 10, 2021. The copyright holder for this preprint (which was not certified by peer review) is the author/funder. All rights reserved. No reuse allowed without permission.

Gd vs dOHb Supplementary Data

Supplementary Figure 1: The perfusion maps using dOHb and Gd in all 6 subjects.

The perfusion maps showcasing similarities of the two different methods, deoxyhemoglobin, and

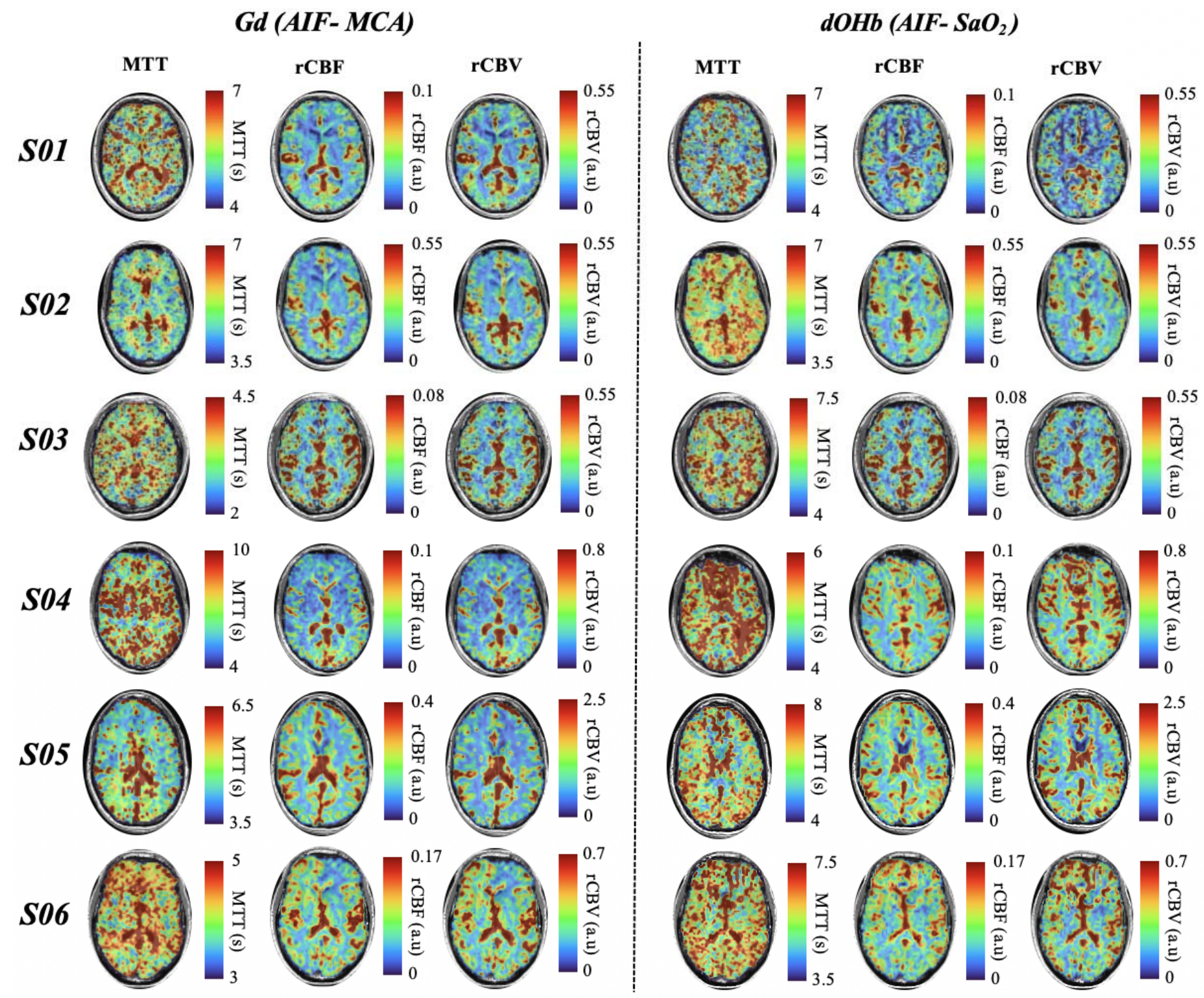

gadolinium in six participants. 


\section{Supplementary Table 1: Comparison of Gd based contrast agent and [dOHb] as} susceptibility contrast agents.

Abbreviations: ([dOHb]) deoxyhemoglobin concentration; ([Gd]) gadolinium concentration; (AIF) arterial input function.

\begin{tabular}{|c|c|c|}
\hline $\begin{array}{l}\text { Performance } \\
\text { characteristic }\end{array}$ & [Gd] & [dOHb] \\
\hline Non-invasive & - Intravascular injection & $\begin{array}{l}\text { YES } \\
\text { - Contrast bolus generated by } \\
\text { inhaling gas }\end{array}$ \\
\hline $\begin{array}{l}\text { High SNR: \# } \\
\text { unpaired } \\
\text { electrons }\end{array}$ & $\begin{array}{l}7 \text { unpaired electrons } \\
\text { - The paramagnetic effect is } \\
\text { several-folds higher inducing } \\
\text { a greater } \Delta \mathrm{T} 2 *\end{array}$ & 4 unpaired electrons \\
\hline $\begin{array}{l}\text { Agent tied to } \\
\text { physiological } \\
\text { environment }\end{array}$ & $\begin{array}{r}\mathrm{NO} \\
-\quad \text { (inert) }\end{array}$ & $\begin{array}{l}\text { YES } \\
\text { - Signal strength proportional to } \\
\text { [dOHb] }\end{array}$ \\
\hline $\begin{array}{l}\text { Contrast } \\
\text { remains } \\
\text { intravascular }\end{array}$ & $\mathrm{NO}$ & YES \\
\hline $\begin{array}{l}\text { Contrast } \\
\text { concentration } \\
\text { known }\end{array}$ & $\begin{array}{l}\text { NO } \\
\text { Enabling blood [Gd] } \\
\text { calculation: } \\
\text { - Known dose } \\
\text { - Known rate of injection } \\
\text { Obscuring blood [Gd] } \\
\text { calculation: } \\
\text { - Saturation of signal loss } \\
\text { possible with excessive } \\
\text { dosing. } \\
\text { Unknown (i) cardiac } \\
\text { output; (ii) dispersion in } \\
\text { venous system; (iii) } \\
\text { extravasation during lung } \\
\text { and heart transit; (iv) } \\
\text { dispersion in heart }\end{array}$ & $\begin{array}{l}\text { YES } \\
\text { - } \text { Precise arterial }[\mathrm{dOHb}] \\
\text { calculated from } \mathrm{P}_{\mathrm{ET}} \mathrm{O}_{2} * \\
\text { - Minimal dispersion in healthy } \\
\text { left ventricle } \\
\text { *Note: Accuracy may be } \\
\text { limited by disease with } \\
\text { right to left shunt in heart } \\
\text { and lungs. }\end{array}$ \\
\hline $\begin{array}{l}\text { Same scale as } \\
\text { tissue curve, }\end{array}$ & $\begin{array}{l}\text { Relation of arterial and } \\
\text { tissue scales uncertain }\end{array}$ & - Intravascular agent only \\
\hline
\end{tabular}


Gd vs dOHb Supplementary Data

\begin{tabular}{|c|c|c|}
\hline $\begin{array}{l}\text { or known } \\
\text { scale factor }\end{array}$ & $\begin{array}{l}\text { - } \mathrm{R} 2 * \text { quadratic dependence on } \\
{[\mathrm{Gd}]}\end{array}$ & \\
\hline $\begin{array}{l}\text { signal } \\
\text { saturation }\end{array}$ & $\begin{array}{l}\text { - Saturates easily at high doses } \\
\text { due to high SNR; phase analysis } \\
\text { may also saturate }\end{array}$ & - Low SNR: Unlikely to saturate \\
\hline $\begin{array}{l}\text { No signal } \\
\text { aliasing }\end{array}$ & - Aliasing if phase shift $>180 \mathrm{deg}^{45}$ & - Phase shift analysis unknown \\
\hline
\end{tabular}

